\title{
Influence of a complex network substrate on reaction-diffusion processes
}

\author{
Lazaros K Gallos and Panos Argyrakis \\ Department of Physics, University of Thessaloniki, 54124 Thessaloniki, Greece \\ E-mail: panos@physics.auth.gr
}

Received 2 October 2006

Published 22 January 2007

Online at stacks.iop.org/JPhysCM/19/065123

\begin{abstract}
We discuss recent work on reaction-diffusion processes that take place on complex networks. The inherent inhomogeneity of the substrate leads to a series of new phenomena, which bear only a small resemblance to the classical results of the field. The annihilation rate is abnormally high in such systems, while at the same time depletion zones are absent in the $\mathrm{A}+\mathrm{A} \rightarrow 0$ reaction and no segregation is observed for the $\mathrm{A}+\mathrm{B} \rightarrow 0$ reaction. These results are mainly attributed to the presence of network hubs and to the small network diameter of such systems.
\end{abstract}

\section{Introduction}

\subsection{Reaction-diffusion}

Reaction-diffusion models are among the most successful and important tools in the field of chemical kinetics. The basic idea is that a number of molecules diffuse in a given space and upon encounter with other molecules a reaction takes place that results in partial or complete annihilation of the reactants and/or to new reaction products. A reaction-limited process can be described by mean-field analysis, while a diffusion-limited model can also be handled efficiently via exact results and computer simulations. The combination of both processes in the same model, though, results in a number of novel and intuitively unexpected features.

Among the simplest processes that can describe a wide range of observed phenomena are the bimolecular annihilation reactions $\mathrm{A}+\mathrm{A} \rightarrow 0$ and $\mathrm{A}+\mathrm{B} \rightarrow 0$. For the one-species reaction, molecules of the same type diffuse and annihilate when they encounter each other. For the two-species reaction, two molecules of different species will annihilate, while if they are of the same type they will just bounce on each other.

Early works [1-3] revealed a behaviour drastically different from the mean field predictions, especially in low dimensions and in fractal structures. Numerical simulations followed that verified some novel effects in these two systems, i.e. the generation of a depletion 
zone for the A+A type [2] and the spatial segregation of the two types of reactants in the $A+B$ type [4]. An enormous amount of research has piled up since then, and the behaviour of these systems has been studied under a plethora of different conditions [5].

Classically, the mean-field prediction for the density $\rho$ of the surviving particles in $\mathrm{A}+\mathrm{A}$ and equimolar $\mathrm{A}+\mathrm{B}$ type reactions is

$$
\frac{1}{\rho}-\frac{1}{\rho_{0}}=k t^{f}
$$

where $f=1, k$ is the rate constant, and $\rho_{0}$ is the particle density at $t=0$. In non-classical kinetics, though, an 'anomalous' behaviour has been observed. In a space with dimensionality $d$ the exponent $f=d / d_{\mathrm{c}}$ for $d \leqslant d_{\mathrm{c}}$, and $f=1$ for $d>d_{\mathrm{c}}$. The upper critical dimension $d_{\mathrm{c}}$ equals 2 for $\mathrm{A}+\mathrm{A}$, and $d_{\mathrm{c}}=4$ for $\mathrm{A}+\mathrm{B}$. Similarly, anomalous behaviour has been observed when the diffusion-reaction process takes place on different geometries, such as on fractals [6] where $f=d_{\mathrm{s}} / d_{\mathrm{c}}$, or on dendrimer structures [7]. In all these cases the limiting value of the exponent is $f=1$, valid even in infinite dimensions.

Recent work, though, revealed that when the substrate of this process has a largely inhomogeneous character, such as a scale-free network, a completely different picture emerges. The exponent $f$ may assume a value that is significantly larger than unity for both reactions, indicating a very efficient mixing process that results in rapid annihilation of the involved species. This unusual behaviour has been attributed to the specific features of this class of networks, and will be reviewed in the current study.

\subsection{Complex networks}

A huge number of networks spanning many disciplines have been shown to have some important common features, such as the small-world property and the scale-free character $[8,9]$. The notion of a network is very wide and can describe any system where certain entities (the network nodes) interact with each other. Each interaction between two nodes results in a link between them. The small-world property refers to the small distance that separates any two nodes in the network compared with the total network size $N$. The scalefree character reveals the large inhomogeneity in the network structure, where the distribution of the number of links $k$ of a node is very wide and is usually described by a power law form: $P(k) \sim k^{-\gamma}$. The exponent $\gamma$ is a network characteristic, with typical values in the range 2.0-4.0. This distribution means that while the majority of the nodes have a small degree $k$ there exist a very small number of nodes (the network 'hubs') that possess a very large connectivity. Despite their rareness, though, these nodes significantly influence almost all static and dynamic properties in a network, compared to a network with a uniform degree distribution.

During the reaction-diffusion process the number of particles $M(t)$ in the system is reduced with time, and we denote with $\rho(t)$ the particle concentration at time $t$. The initial particle concentration is $\rho_{0}$ and the total number of nodes in the network is $N$. A number of $N_{0}=\rho_{0} N$ particles are placed on randomly selected nodes (in simulations of this work $\rho_{0}=0.5$ ). Then, one particle is randomly chosen and one of the neighbouring nodes where this particle is located is selected. If this new node is empty, the particle moves and occupies its new position. If this node is already occupied, then for the $\mathrm{A}+\mathrm{A}$ reaction the two particles annihilate and are immediately removed from the system. For the $\mathrm{A}+\mathrm{B}$ reaction the two particles annihilate only if they belong to different species, while if they are of the same type, the chosen particle remains in its original position. Time is advanced inversely proportionally to the current number of particles, by $1 / M(t)$. This procedure is repeated by continuously selecting, moving and (possibly) annihilating particles. 

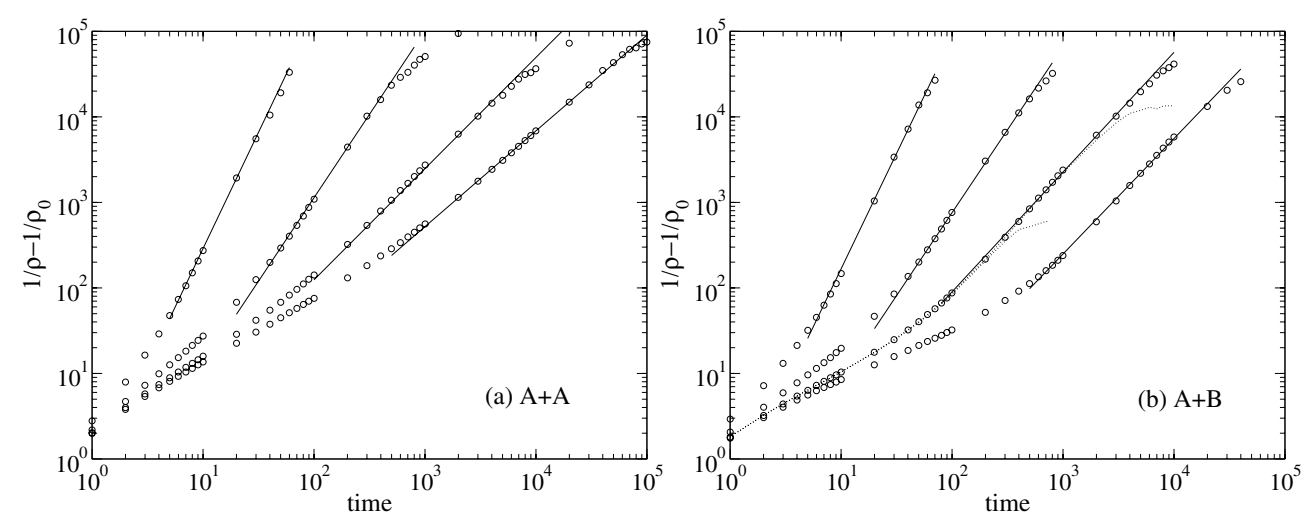

Figure 1. Plots of the reaction progress $1 / \rho-1 / \rho_{0}$, as a function of time for (a) the $\mathrm{A}+\mathrm{A} \rightarrow 0$ reaction and (b) the $\mathrm{A}+\mathrm{B} \rightarrow 0$ reaction on scale-free networks of (left to right) $\gamma=2.0,2.5,3.0$, and 3.5. The initial density was $\rho_{0}=0.5$ and $\rho_{0}=0.25$ respectively. All results correspond to networks of $N=10^{6}$ nodes, except for the dotted lines in (b), where we present results for systems of $N=10^{4}$ and $10^{5}$ nodes. The symbols represent the simulation data, while continuous lines are the asymptotic best-fit lines.

\section{Reaction rates}

\subsection{Simulations}

The first indication that the properties of a reaction-diffusion process on scale-free networks present some unique features was numerical [10, 11]. Direct simulations of the particle concentration $\rho(t)$ (figure 1) revealed that for a very wide $\gamma$ range the evolution of $\rho(t)$ follows a power-law, with two distinct regimes. The early regime has a slope less than unity, but asymptotically the reaction rate increases to a remarkably higher slope. The location of the crossover point, also, increases with $\gamma$, while the reaction rate is much faster for lower $\gamma$ values, where the hubs in the network are stronger. The asymptotic behaviour is found to be quite stable. In figure 1(b) and for $\gamma=3.0$ results are presented for three different sizes $\left(N=10^{4}, 10^{5}\right.$, and $\left.10^{6}\right)$. It is obvious that the asymptotic slope is extended and becomes more prominent with increasing network size, before finally the finite-size effects settle in.

Calculation of the asymptotic slopes for the curves in figure 1 (corresponding to the exponent $f$ of equation (1)) are presented in figure 2, as a function of $\gamma$. The slopes of the $\mathrm{A}+\mathrm{A}$ and the $\mathrm{A}+\mathrm{B}$ types seem to be close to each other (with the latter slightly larger), but in all cases the slope is greater than unity. The exponent $f$ can acquire very large values ( $f=2.75$ for $\gamma=2$ ) and is a monotonically decreasing function of $\gamma$. This is a remarkably different behaviour, since, as already stated, on all other geometries studied in the literature the exponent $f$ is lower than unity. On scale-free networks the value $f=1$ seems to be only reached asymptotically for networks with large $\gamma$ values.

\subsection{Theory}

Following these findings, Catanzaro et al [12] presented analytical mean-field results for the $\mathrm{A}+\mathrm{A}$ reaction, and very recently Weber and Porto [13] extended these results for the A + B reaction. Both these works consider networks created via the uncorrelated configuration model (UCM) [14]. The main difference between the standard configuration model (CM) and UCM is the fact that no correlations exist in the node connectivity (in this context, correlations exist when the average connectivity of a node's neighbours depend on its degree $k$ ). 


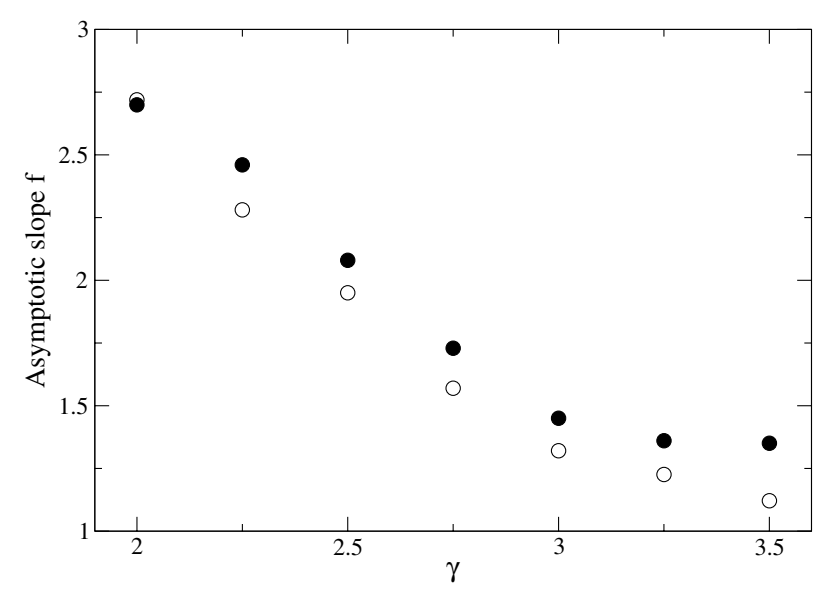

Figure 2. Asymptotic reaction rates (slopes of the lines in figure 1), as a function of $\gamma$, for the $\mathrm{A}+\mathrm{A} \rightarrow 0$ reaction (open symbols) and the $\mathrm{A}+\mathrm{B} \rightarrow 0$ reaction (filled symbols).

The basic steps of the analysis in [12] are as follows: for the A + A reaction, the starting point is the master equation for the occupation variable $n_{i}(t)$ of a node $i$ at time $t$ :

$$
n_{i}(t+\mathrm{d} t)=n_{i}(t) \eta(\mathrm{d} t)+\left[1-n_{i}(t)\right] \xi(\mathrm{d} t),
$$

where $n_{i}(t)$ can be zero or unity, if the node is empty or occupied by an A particle, respectively, while $\eta(\mathrm{d} t)$ and $\xi(\mathrm{d} t)$ are dichotomous random variables that represent the probability for a site to retain or modify its current status (empty or occupied). Averaging over all nodes and summing for all configurations we get the exact result

$$
\frac{\mathrm{d} \rho_{i}(t)}{\mathrm{d} t}=-\rho_{i}(t)+\sum_{j} \frac{a_{i j}}{k_{j}}\left(\rho_{j}(t)-2 \rho_{i j}(t)\right),
$$

where $a_{i j}$ is the adjacency matrix, and the particle concentrations are defined as $\rho_{i}(t) \equiv\left\langle n_{i}(t)\right\rangle$ and $\rho_{i j}(t) \equiv\left\langle n_{i}(t) n_{j}(t)\right\rangle$. The following analysis assumes that all same-degree nodes are equivalent to each other and also that the network is uncorrelated, i.e. the conditional probability $P\left(k^{\prime} \mid k\right)$ is independent of $k$. The final form for the density is then found to be

$$
\frac{\mathrm{d} \rho(t)}{\mathrm{d} t}=-2\left(\frac{\rho(t)}{\langle k\rangle}\right)^{2} \sum_{k} \frac{k^{2} P(k)}{1+2 k \rho(t) /\langle k\rangle} .
$$

For homogeneous networks, where the degree distribution of the nodes is very narrow, a Taylor expansion for small particle densities $\rho$ and large times yields the mean field behaviour

$$
\frac{1}{\rho(t)}-\frac{1}{\rho_{0}} \simeq \frac{2\left\langle k^{2}\right\rangle}{\langle k\rangle^{2}} t
$$

with an asymptotic exponent $f=1$. For highly inhomogeneous substrates, though, such as scale-free networks where the degree distribution follows $P(k) \sim k^{-\gamma}$, the predicted behaviour for the UCM model is [12]

$$
\frac{1}{\rho(t)} \sim \begin{cases}t^{1 /(\gamma-2)}, & 2<\gamma<3 \\ \ln (t), & \gamma=3 \\ t, & \gamma>3 .\end{cases}
$$

If we also take into account finite-size effects, then all the above exponents converge to the mean-field value $f=1$, after a finite crossover time. Thus, it is possible that in numerical simulations the asymptotic behaviour of $f>1$ is masked by the mean-field behaviour. 
The importance of the hubs can also be deduced from the same analysis, where it is shown that the concentration of particles on hubs with degree $k>\langle k\rangle / 2 \rho\left(t^{*}\right)$ for times $t<t^{*}$ is constant and equal to $\left\langle\rho_{k}(t)\right\rangle \simeq 0.5$. This means that the hubs are easily accessible for the particles and with high probability they are both occupied and surrounded by particles. Thus, there is an almost constant cycle of occupation and annihilation on the hubs, which results in the local concentration $\rho_{k}(t)$ alternating between unity and zero. These 'dynamical' hubs are responsible for the initial rapid concentration decay. As time advances, though, and the total concentration $\rho(t)$ decreases, the number of dynamical hubs in the system diminishes, until finally they effectively disappear and the decay becomes linear with time.

The case of $\mathrm{A}+\mathrm{B} \rightarrow 0$ [13] was treated with similar arguments and was shown to be quite similar to that of $\mathrm{A}+\mathrm{A} \rightarrow 0$. Weber and Porto showed analytically the existence of 'jamming' for early times and relatively high concentrations, but only for small-degree nodes. Intuitively, this behaviour is similar to segregation of like particles in lattices, since a particle that is, for example, located in the edge of a network branch and has neighbours of the same type cannot be reached by an unlike particle before its neighbours are annihilated. For the hubs this situation is much more unlikely to appear because there is a very small probability that the large number of a hub's neighbour nodes will all be occupied by the same type of particles. The 'dynamical' hubs described above are, thus, still present for the $\mathrm{A}+\mathrm{B}$ reaction, with the difference that now for one-third of the time they are empty, they are occupied one-third of the time by a particle A, and one-third by a particle B. This also supports the idea that the existence of the hubs tends to make the two reactions more similar to each other in scale-free networks rather than in homogeneous space e.g. on lattices.

The above analytic results confirm the main results of the numerical simulations with the CM model, but the predicted exponent values are not the same. The problem is a bit more complicated, since it seems that the numerical exponent values depend on the details of the network generation method. In the following section, we discuss the influence of the choice between UCM and CM, as well as another important factor, namely the minimum possible degree $k_{\min }$ for a node in the network.

\section{Importance of the network generation model}

We can combine UCM or CM with values $k_{\min }=1$ and $k_{\min }=2$, in order to have a better understanding of how each of these factors influences the system evolution. Results for the time evolution of the particle density in all these four possible cases are shown in figure 3 . All four curves in the case of $\gamma=2.5$ (figure 3(a)) behave differently from each other. The CM and UCM models clearly yield different results, but even within UCM or CM the curves for $k_{\min }=1$ and $k_{\min }=2$ deviate from each other. The main difference is that the crossover to the power law behaviour for $k_{\min }=1$ appears later in time, and the asymptotic mean-field behaviour $(f=1)$ also exhibits itself roughly one decade later.

The case of $\gamma=3.0$ (figure 3(b)) is simpler. By construction, for this $\gamma$ value the two models (CM and UCM) are expected to coincide. This coincidence is shown to be valid in the figure. However, the results for $k_{\min }=1$ are still different from the results for $k_{\min }=2$. The crossover to the power-law behaviour is not as prominent as in the case of $k_{\min }=1$.

A more careful analysis of the slopes in figure 3 [11] revealed that for $\gamma=2.5$ the only case which is close to a linear asymptotic behaviour is that of the UCM model and $k_{\min }=2$, and even then, the result is not entirely satisfactory. Additionally, in almost all cases there exists a regime in which a power-law behaviour is clearly identifiable with a slope $f>1$, whose exact value varies with the model and $k_{\min }$ value used. The validity of the power law, though, lies in 


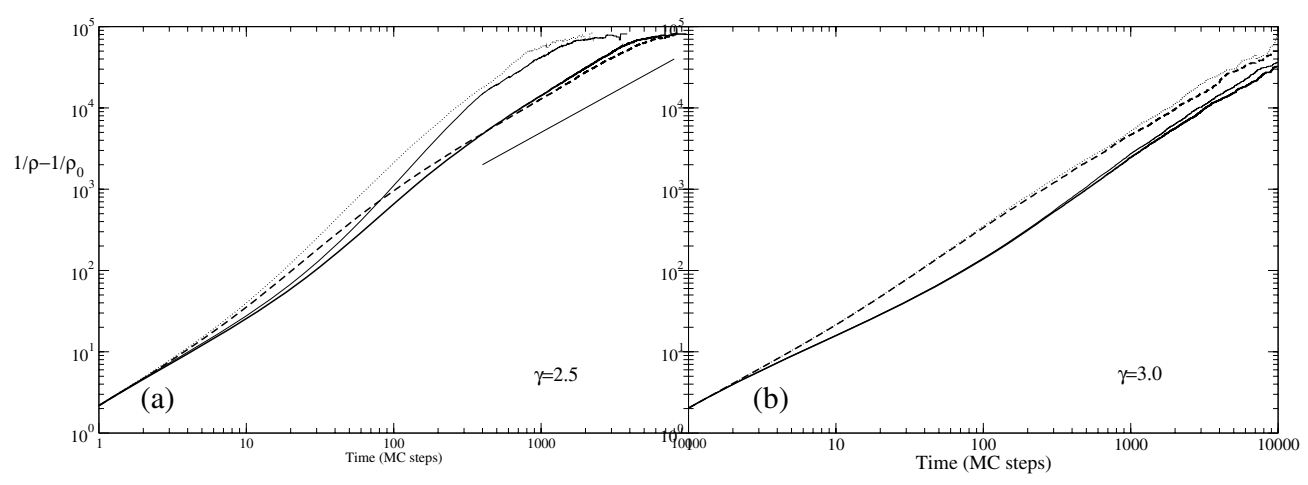

Figure 3. Plot of the reaction progress $1 / \rho-1 / \rho_{0}$, as a function of time for the $\mathrm{A}+\mathrm{A} \rightarrow 0$ reaction on scale-free networks of (a) $\gamma=2.5$, and (b) $\gamma=3.0$. Results correspond to CM (thin lines) and UCM (thick lines) models, with $k_{\min }=1$ (solid lines) and $k_{\min }=2$ (dashed lines). The initial density was $\rho_{0}=0.5$.

a narrower time range for $k_{\min }=2$, and especially from the UCM model. The slopes of the curves for $k_{\min }=1$ are consistently larger compared to the slopes of the $k_{\min }=2$ curves.

Similar results were also found for $\gamma=3.0$, where the analysis predicts a logarithmic correction $(1 / \rho \sim t \ln t)$. In general, it is not easy to distinguish between this form and a power law curve. However, it can be seen that for $k_{\min }=2$ there is an excellent agreement, but this function fails to reliably describe the results for $k_{\min }=1$.

The most probable explanation for the difference in the results for the varying $k_{\min }$ values is that the local environment in the case of $k_{\min }=1$ is remarkably different from when $k_{\min }=2$. Although $k_{\min }=2$ is known to yield one giant connected cluster [15], its structural characteristics seem to be modified, basically because of the tree-like features (many nodes are connected to just one neighbour). Bottlenecks and revisitations are, thus, more frequent than in the case where a larger lower cut-off is used.

\section{Absence of depletion zones and segregation}

The typical behaviour of reaction-diffusion models in regular geometries, such as lattices or homogeneous space, is the generation of a depletion zone for the $\mathrm{A}+\mathrm{A}$ type and the spatial segregation of the two types of reactants in the A + B type. Intuitively, it is easy to understand that for the $\mathrm{A}+\mathrm{A}$ reaction the A particles that are close to each other tend to quickly annihilate, leaving at longer times a large particle-free zone around each surviving particle. For the A + B type, reaction incidents between A and B take place only on the interface between blocks of A or B particles. These blocks tend to increase in size with time, resulting in segregation of the involved species.

When the substrate is scale free, though, the inverse picture emerges. For the $\mathrm{A}+\mathrm{A}$ reaction, most of the particles tend to cluster around the network hubs, which can also account for the rapid decrease of the concentration $\rho(t)$. As time advances, this clustering increases, leading to an even faster $\rho(t)$ decay. This behaviour is in sharp contrast with the idea of a depletion zone, since we can see that even in low concentrations particles are never too far from each other. Similarly, for the A + B reaction it was shown that there is almost no species segregation. Because of the network inhomogeneity there is a very low probability for a large number of particles to form a close formation that cannot be 'penetrated' by the other species. Thus, the segregation effect is also absent in these systems. 

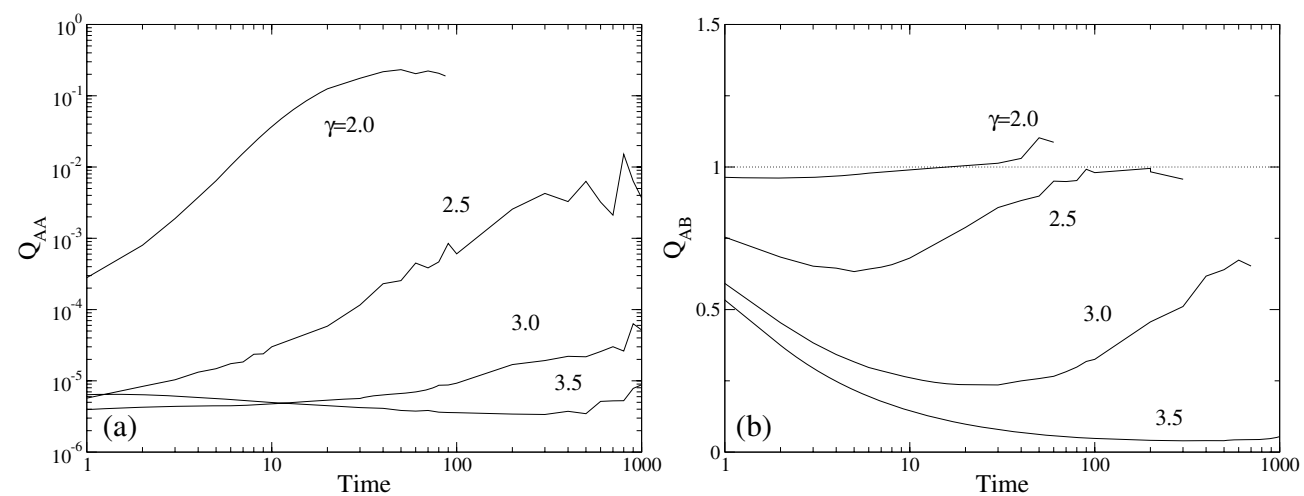

Figure 4. (a) $\mathrm{A}+\mathrm{A}$ reaction: percentage $Q_{\mathrm{AA}}$ of contacts between A particles over the total number of possible contacts as a function of time. (b) $\mathrm{A}+\mathrm{B}$ reaction: percentage of $\mathrm{AB}$ contacts over $(\mathrm{AA}+\mathrm{BB})$ contacts as a function of time. The line at $Q_{\mathrm{AB}}=1$ corresponds to complete mixing. The $\gamma$ values are as marked.

We can quantify these properties by calculating the number of close contacts between particles as a function of time. For the $\mathrm{A}+\mathrm{A}$ reaction we measure the ratio $N_{\mathrm{AA}}(t)$ of close contacts over the total possible number of contacts at time $t$

$$
Q_{\mathrm{AA}}(t)=\frac{N_{\mathrm{AA}}(t)}{M(t)(M(t)-1)} .
$$

A value of $Q_{\mathrm{AA}}=1$ shows that all particles form one block, while smaller values indicate the existence of a depletion zone. The numerical results for $Q_{\mathrm{AA}}$ in figure 4(a) show that, contrary to this, the value of this ratio increases with time.

For the $\mathrm{A}+\mathrm{B}$ reaction we use the ratio $Q_{\mathrm{AB}}(t)$ of contacts between unlike particles over the number of contacts between like particles:

$$
Q_{\mathrm{AB}}(t)=\frac{N_{\mathrm{AB}}(t)}{N_{\mathrm{AA}}(t)+N_{\mathrm{AB}}(t)} .
$$

Segregation is denoted by a value close to $Q_{\mathrm{AB}}=0$, while a value of $Q_{\mathrm{AB}}=1$ indicates complete mixing of the two species. Figure 4(b) supports the idea of asymptotic mixing, at least for networks with exponents $\gamma<3.5$.

\section{Probability of annihilation on a given node}

Due to the large inhomogeneity of a scale-free network, its nodes present very different local properties. In the following, we present some results on the $\mathrm{A}+\mathrm{A}$ reaction where we focus on the nodes where the annihilation events take place.

After $t=100$ steps, when the particle concentration has diminished to small values, we can calculate the percentage of annihilation events $F_{\text {ann }}(k)$ that took place on a node with $k$ links. Figure 5 shows $F_{\text {ann }}(k)$ as a function of $k$. The four curves in the figure are the result of logarithmic binning at degrees $k>10$.

In a mean-field treatment of a network with no degree-degree correlation we expect that the number of events would be proportional to the total number of links leading to a $k$-node, i.e.

$$
F_{\text {ann }}(k) \sim k P(k) \sim k^{1-\gamma}
$$




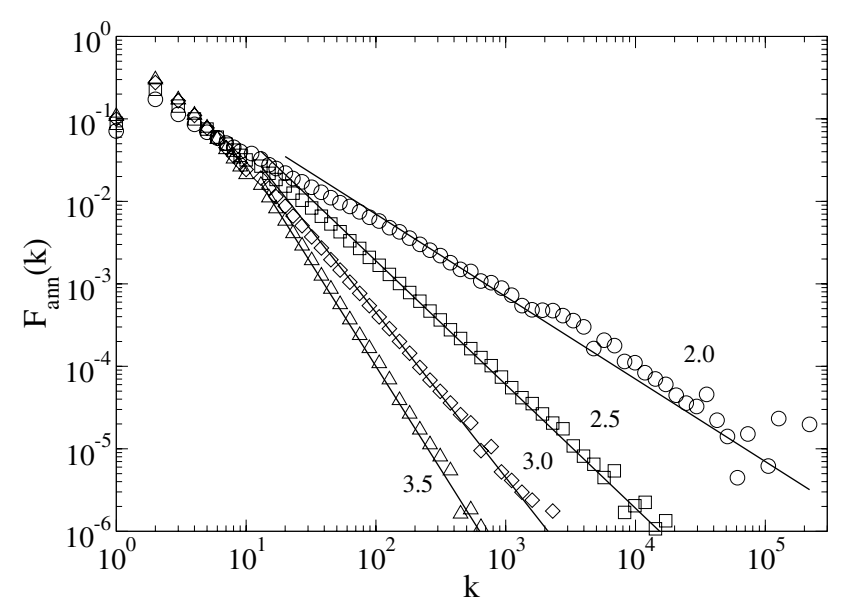

Figure 5. Probability, $F_{\text {ann }}(k)$, for an annihilation event to take place on a node with $k$ links as a function of $k$, during a process of $t=100$ steps. Symbols are averages over 100 scale-free networks with $\gamma=2.0,2.5,3.0$ and 3.5 (top to bottom, shown in the plot). The data have been logarithmically binned for $k>10$. Lines represent equation (9), with the corresponding exponent $1-\gamma$ for each case.

The exponent $1-\gamma$ is verified in all four cases, as can be seen in figure 5 , for the intermediate to large $k$ regime. The result of equation (9) and the presented curves show that even though most of the events take place on low- $k$ nodes, which comprise most of the network, the probability for annihilation at the hubs is significantly larger than their relative appearance in a network. The behaviour seems also not to be influenced by any degree-degree correlation in the network.

The importance of the hubs can be made explicit if we ask what the average number of events $R(k)$ is on a single node with $k$ links, i.e.

$$
R(k)=\frac{F_{\mathrm{ann}}(k)}{P(k)} \text {. }
$$

According to equation (9) we expect a linear increase with the number of links $k$, since $R(k) \sim k^{1-\gamma} / k^{-\gamma} \sim k$. This behaviour is verified in figure 6 , at least for large $k$ values. The slope of $\gamma=2$ seems to be slightly different from the other curves, but this can be attributed to the increasing number of hubs at this $\gamma$ value, where the network has a very small diameter. The curves in figure 2 span many decades on the $y$-axis, manifesting a very different role of nodes with different degrees.

In a typical walk of $t=100$ steps about $2 \times 10^{5}$ events are observed. If we focus on a particular node with a small degree we see that there is much less than one annihilation event occurring on this node during the process. In contrast, more than $10^{4}$ events occur at the hubs having $k \simeq 10^{5}$ links. We, thus, conclude from figures 4 and 5 that a hub is a much more active element in a network than any other node, although the majority of events still occur over the large number of low- $k$ nodes.

The results until now concerned the entire process after averaging over the first $t=100$ steps. We now turn to the time evolution of the diffusion-reaction mechanism. In figure 7(a) we can see how $F_{\text {ann }}(k=1)$ varies with time. For all $\gamma$ values the probability starts initially with a certain value and decreases monotonically with time before stabilizing to a much lower value. For $\gamma=3$ and $\gamma=3.5$, where hubs are not very strongly connected, the probability goes down to a value of 0.02 even for the longer times displayed. For $\gamma=2$ and $\gamma=2.5$, though, the probability that an annihilation occurs on a node with $k=1$ practically vanishes. 


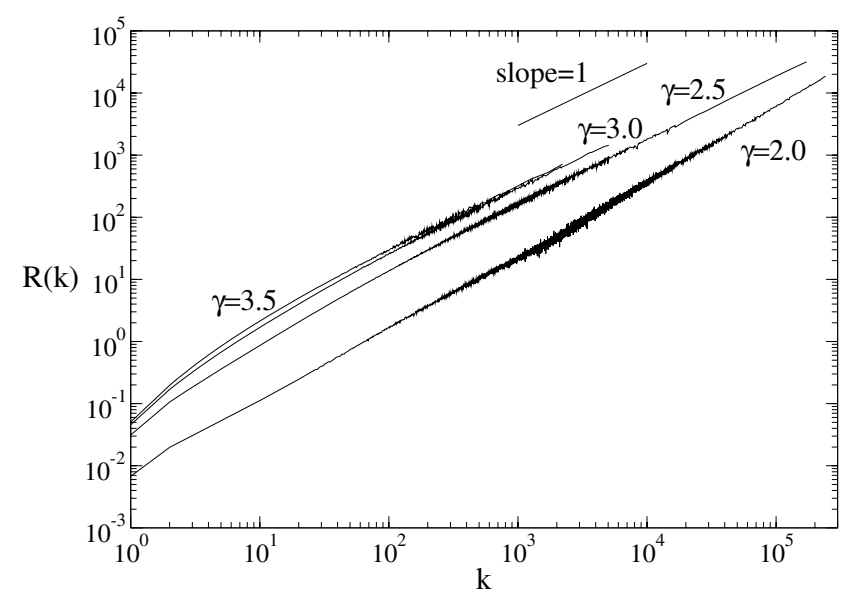

Figure 6. Average number of annihilation events per node, $R(k)$, with $k$ links as a function of $k$, during a process of $t=100$ steps. Curves are averages over 100 scale-free networks with $\gamma=2.0$, 2.5, 3.0 and 3.5, of $N=10^{6}$ nodes each.
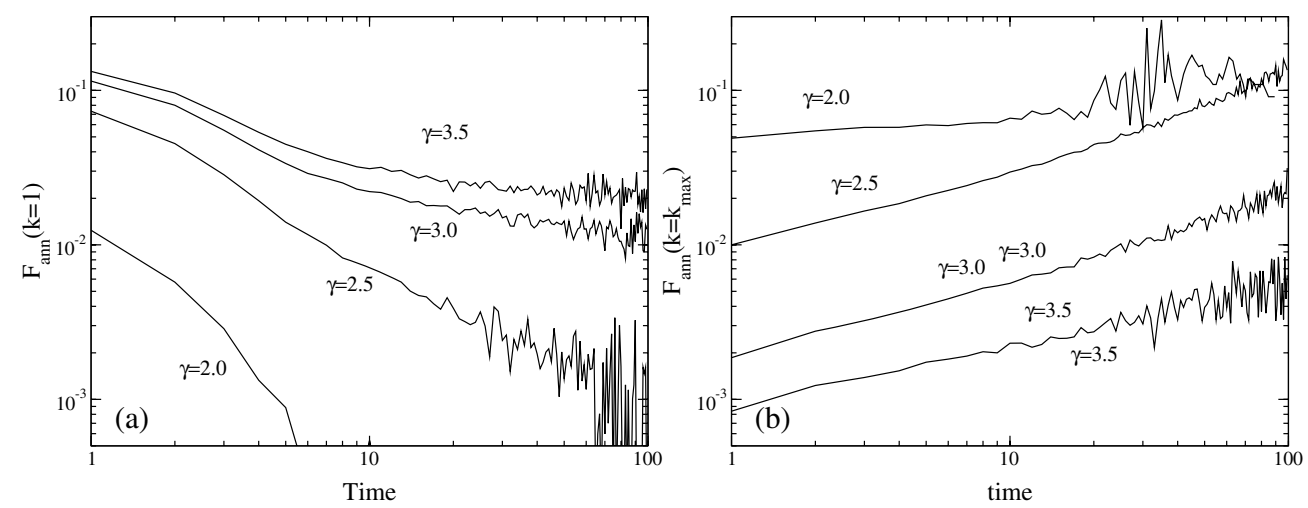

Figure 7. Time evolution of the average probability (a) $F_{\text {ann }}(k=1)$ and (b) $F_{\text {ann }}\left(k=k_{\max }\right.$ ), for networks with varying $\gamma$ exponents.

This picture is reversed when we monitor the probability $F_{\text {ann }}\left(k_{\max }\right)$, i.e. the fraction of events taking place on the single most connected node of the network during the time interval $[t, t+1]$ over the total number of events that occurred during the same interval. Figure 7(b) suggests that now $F_{\text {ann }}\left(k_{\max }\right)$ starts with low values but continuously increases as time evolves. Note that for networks with $\gamma<3$ more than $10 \%$ of the events at long times take place on this node alone.

\section{Summary}

The study of dynamical processes on unusual geometries and/or under unusual conditions has recently attracted a lot of interest. Surprising new findings provide new challenges to the otherwise well studied reaction-diffusion mechanisms. For example, Zumofen et al [16] have studied bimolecular reactions for Levy walks, where under given conditions for $d=3$ no segregation is observed. The results for scale-free networks, briefly reviewed above, are also counter-intuitive. Reactions between three species were studied on both square lattices [17] and 
scale-free networks [18]. Migration-driven growth has also been shown to behave differently on homogeneous [19] and non-homogeneous space [20].

The presented results strongly suggest that the reaction process follows a very different path than the ones intuitively expected from our experience with homogeneous space. The absence of the usual kinetic effects of depletion and segregation leads to a very efficient mixing of the reactants, which in turn results in a significantly accelerated reaction rate. The particles are 'attracted' from the few nodes with the largest degree and the reactions $\mathrm{A}+\mathrm{A} \rightarrow 0$ and $\mathrm{A}+\mathrm{B} \rightarrow 0$ behave quite similarly to each other.

\section{Acknowledgments}

This work was supported by a NEST/PATHFINDER project DYSONET/012911 of the EC, and also by a project of the Greek GGET in conjunction with ESF in the frame of international organizations.

\section{References}

[1] Ovchinnikov A A and Zeldovich Ya B 1978 Chem. Phys. 28215

[2] Torney D C and McConnell H E 1983 J. Phys. Chem. 871941

[3] Torney D C and McConnell H E 1983 Proc. R. Soc. A 387147

[4] Toussaint D and Wilczek F 1983 J. Chem. Phys. 782642

[5] ben-Avraham D and Havlin S 2000 Diffusion and Reactions in Fractals and Disordered Systems (Cambridge: Cambridge University Press)

[6] ben-Avraham D and Havlin S 1987 Adv. Phys. 36695

[7] Argyrakis P and Kopelman R 2000 Chem. Phys. 261391

[8] Albert R and Barabasi A-L 2002 Rev. Mod. Phys. 7447

[9] Dorogovtsev S N and Mendes J F F 2002 Adv. Phys. 511079

[10] Gallos L K and Argyrakis P 2004 Phys. Rev. Lett. 92138301

[11] Gallos L K and Argyrakis P 2005 Phys. Rev. E 72017101

[12] Catanzaro M, Boguñá M and Pastor-Satorras R 2005 Phys. Rev. E 71056104

[13] Weber S and Porto M 2006 Phys. Rev. E 74046108

[14] Catanzaro M, Boguñá M and Pastor-Satorras R 2005 Phys. Rev. E 71027103

[15] Newman M E J, Strogatz S H and Watts D J 2001 Phys. Rev. E 64026118

[16] Zumofen G, Klafter J and Shlesinger M F 1996 Phys. Rev. Lett. 772830

[17] Kim K, Chang K H and Kong Y S 1999 J. Phys. Soc. Japan 681450

[18] Kim K, Chang K H, Yum M K, Choi J S and Odagaki T 2005 Preprint cond-mat/0503674

[19] Leyvraz F and Redner S 2002 Phys. Rev. Lett. 88068301

[20] Ke J, Lin Z, Zheng Y, Chen X and Lu W 2006 Phys. Rev. Lett. 97028301 